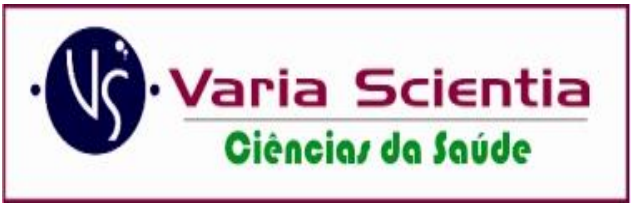

e-ISSN 2446-8118

\title{
ATUAÇÃO DO RESIDENTE DE ENFERMAGEM NO ENFRENTAMENTO À COVID-19 NO MUNICÍPIO DE CASCAVEL/PR: RELATO DE EXPERIÊNCIA
}

\author{
NURSE RESIDENT'S PERFORMANCE FACING THE COVID-19 IN CASCAVEL/PR \\ EXPERIENCE REPORT
}

\author{
DESEMPEÑO DEL RESIDENTE DE ENFERMERÍA EN EL ENFRENTAMIENTO A LA \\ COVID-19 EN LA CIUDAD DE CASCAVEL/PR: RELATO DE EXPERIENCIA
}

Gilson Fernandes da Silva ${ }^{1}$

Beatriz Talluly Bespalhok ${ }^{2}$

Fernanda Cristina Mucelini ${ }^{3}$

Pamela Bodaneze ${ }^{4}$

Yasmin Luisa Dengo Lombardo ${ }^{5}$

RESUMO: Diante do contexto de pandemia e do enfrentamento à COVID-19, este estudo tem por objetivo revelar as experiências vivenciadas pelos residentes de enfermagem do programa de residência multiprofissional em saúde da família, durante o período de enfrentamento à COVID-19. Trata-se de um relato de experiência, de abordagem descritivo-reflexiva, baseado na atuação e em ações desenvolvidas pelos residentes de enfermagem, entre os meses de março a outubro de 2020 , no município de Cascavel/PR. O enfrentamento da pandemia permitiu compreender que, mesmo na adversidade, a equipe de saúde foi capaz de se reinventar e reorganizar ações dos programas para garantir a atenção à saúde, tanto aos usuários como às demandas pertinentes aos protocolos da Estratégia da Saúde da Família, além daqueles apresentados pela COVID-19. Ficou evidente a importância do papel do residente enquanto componente da equipe, da relevância para o cuidado da população, considerando que houve déficit de profissionais na assistência. Essa experiência proporcionou aos residentes a atuação como enfermeiros, tanto na assistência direta quanto no gerenciamento das atividades dos serviços de saúde. Dessa forma, as articulações, desenvolvidas pelos residentes de enfermagem, oportunizaram o relacionamento entre as diferentes áreas profissionais da saúde. Com a pandemia, foi possível ampliar a visão acerca do cuidado, identificando a importância das outras categorias profissionais, além de possibilitar a socialização do conhecimento apreendido nesse contexto da pandemia da COVID-19, como agentes disseminadores de informações nos diversos espaços do território.

${ }^{1}$ Secretaria Municipal de Saúde de Cascavel. Escola de Saúde Pública Municipal. Enfermeiro. Gerente da Escola de Saúde Pública de Cascavel/PR. Coordenador e Tutor do Programa de Residência Multiprofissional em Saúde da Família - Cascavel/PR. Secretaria Municipal de Saúde de Cascavel/PR/Brasil. Mestre em Biociências e Saúde pela UNIOESTE.

2 Secretaria Municipal de Saúde de Cascavel/PR/Brasil. Enfermeira. Residente do Programa de Residência Multiprofissional em Saúde da Família - Cascavel/PR. Secretaria Municipal da Saúde de Cascavel/PR/Brasil.

3 Secretaria Municipal de Saúde de Cascavel/PR/Brasil. Enfermeira. Residente do Programa de Residência Multiprofissional em Saúde da Família - Cascavel/PR. Secretaria Municipal de Saúde de Cascavel/PR/Brasil.

4 Secretaria Municipal de Saúde de Cascavel/PR/Brasil. Enfermeira. Residente do Programa de Residência Multiprofissional em Saúde da Família - Cascavel/PR. Secretaria Municipal de Saúde de Cascavel/PR/Brasil.

5 Secretaria Municipal de Saúde de Cascavel/PR/Brasil. Enfermeira. Residente do Programa de Residência Multiprofissional em Saúde da Família - Cascavel/PR. Secretaria Municipal de Saúde de Cascavel/PR/Brasil. 
DESCRITORES: Pandemia; Internato e Residência; Enfermagem.

\begin{abstract}
In the context of the COVID-19 pandemic, this study reveals the experiences of nursing residents of the multi professional residency program in family health, during the period of confrontation with COVID-19. This is an experience report, of descriptive reflexive approach, based on the performance delivered by nursing residents, between March and October 2020, in the Cascavel / PR - BR. The fight against the pandemic showed that, even in adversity, the health service could reinvent and reorganize actions to ensure health care, both to users and to the protocol demands of the Family Health Strategy, besides those presented by COVID-19. The importance of the resident as part of a team and its relevance to population care, became evident considering the deficit of professionals. This experience allowed the residents to act as nurses, both in direct assistance and in the health services management activities. Thus, the coordination, developed by the nursing residents, improved the relationship between the different professional's areas of health. Due to the pandemic, it was possible to broaden the understanding about care, identifying the importance of other professional categories, in addition to enabling the sharing of knowledge acquired in the context of the COVID-19 pandemic, as agents disseminating information in different environments.
\end{abstract}

DESCRIPTORS: Pandemic; Internship and Residence; Nursing.

RESUMEN: Dado el contexto de la pandemia y el enfrentamiento a la COVID-19, este estudio tiene como objetivo revelar las experiencias vividas por enfermeras residentes del programa de residencia multiprofesional en salud familiar, durante el período de enfrentamiento con COVID-19. Es un relato de experiencia, con enfoque descriptivo-reflexivo, basado en el desempeño y acciones desarrolladas por residentes de enfermería, entre los meses de marzo a octubre de 2020, en la ciudad de Cascavel/PR. El enfrentamiento a la pandemia nos permitió entender que, aun en la adversidad, el equipo de salud supo reinventarse y reorganizar las acciones de programas que aseguran el cuidado de la salud, tanto a los usuarios como a las demandas relevantes a los protocolos de la Estrategia de Salud de la Familia, además de los presentados por la COVID-19. Se evidenció la importancia del papel del residente como parte del equipo, de su relevancia para el cuidado de la población, considerando que hubo un déficit de profesionales en la asistencia. Esta experiencia permitió a los residentes trabajar como enfermeros, tanto en la asistencia directa como en la gestión de las actividades del servicio de salud. Así, las articulaciones, desarrolladas por residentes de enfermería, hicieron posible la relación entre las diferentes áreas profesionales de la salud. Con la pandemia, se logró ampliar la visión sobre el cuidado, reconociendo la importancia de otras categorías profesionales, además de posibilitar la socialización de los conocimientos aprendidos en este contexto de la pandemia COVID-19, como agentes difusores de información en los distintos espacios del territorio.

DESCRIPTORES: Pandemia; pasantía y residencia; enfermería.

\section{INTRODUÇÃO}

Diante do surgimento, em dezembro de 2019, de diversos casos de pneumonia de origem desconhecida, na cidade de Wuhan, província de Hubei, China, e após análise de material genético, constatou-se tratar de um novo Coronavírus, pertencente à família de betacoronavírus, atualmente, denominado
SARS-CoV-2 (do inglês Severe Acute Respiratory Syndrome Coronavirus 2). ${ }^{1}$

A infecção por SARS-CoV-2, também conhecida por Covid-19, pode variar de casos assintomáticos a casos graves. Os sintomas leves assemelham-se aos de um resfriado comum, podendo causar febre, fadiga, coriza e tosse seca; nos casos mais graves, pode evoluir para dispneia e a Síndrome Respiratória Aguda Grave (SRAG). ${ }^{2}$ 
Rapidamente, espalhou-se a outros continentes, afetando países, como Canadá, Estados Unidos e Brasil. Em 30 de janeiro de 2020, a doença foi declarada como estado de emergência de saúde pública global e, posteriormente, em 30 de março de 2020, foi considerada uma pandemia. ${ }^{3}$

No Brasil, a Portaria $\mathrm{n}^{\circ} 188$, de 03 de março de 2020, declarou emergência de saúde pública de importância nacional, em decorrência da infecção humana pelo novo Coronavírus. ${ }^{3} \mathrm{O}$ primeiro caso de COVID-19 foi diagnosticado no dia 26 de fevereiro, na cidade de São Paulo - SP. A transmissão comunitária de COVID-19, no país, foi declarada após a publicação da Portaria $\mathrm{n}^{\circ}$ 454, de 20 de março de $2020 .^{4}$

Com base nesses marcos, as ações foram repassadas para todos os níveis de atenção, a fim de que norteassem os fluxos de atendimento e controle da transmissibilidade do vírus. Os amparos legislativos partiram da Lei $\mathrm{N}^{\mathrm{o}} 13.979$, de 6 de fevereiro de 2020, que dispõe sobre as medidas para enfrentamento da emergência de saúde pública de importância internacional, decorrentes do Coronavírus, responsável pelo surto de $2019 .^{5}$

No que compete ao nível municipal, essas ações foram baseadas no Protocolo de Manejo Clínico do Coronavírus para Atenção Primária, documento que definiu as medidas a serem adotadas no atendimento de casos suspeitos. Esse protocolo foi seguido por todas as Unidades Básicas de Saúde (UBS) e Unidades de Saúde da Família (USF), de maneira que os profissionais de saúde foram capacitados para identificar o curso clínico da infecção, com base no histórico relatado pelo paciente, nos sinais e sintomas clínicos que ele apresentava. ${ }^{1}$

É preciso considerar que a Atenção Primária à Saúde (APS) é o primeiro nível de atenção em saúde e se caracteriza por um conjunto de ações, no âmbito individual e coletivo, que abrange a promoção e a proteção da saúde, a prevenção de agravos, o diagnóstico, o tratamento, a reabilitação, a redução de danos e a manutenção da saúde. Tudo isso com o objetivo de desenvolver uma atenção integral, que impacte positivamente a situação de saúde das coletividades. ${ }^{6}$
Relato de Experiência

Ademais, (APS) é a principal porta de entrada do Sistema Único de Saúde (SUS). Fazendo parte da APS, a Estratégia Saúde da Família (ESF) visa à reorganização desse modelo no país, de acordo com os princípios e diretrizes do SUS. ${ }^{6}$

Nesse contexto, figuram os programas de residência multiprofissional em saúde da família, que têm por objetivo qualificar, especializar e capacitar enfermeiros, do ponto de vista ético, político e técnico-científico. É uma modalidade de formação considerada de excelência com o intuito de contribuir ao processo de atuação desses profissionais, com ênfase na saúde da família, sendo norteados por estratégias pedagógicas, capazes de utilizar e promover cenários de aprendizagem, configurados nas Redes de Atenção à Saúde (RAS). Assim, a ESF é o espaço privilegiado, visto que incorpora os profissionais para a qualificação na prática cotidiana dos serviços de saúde, além de possibilitar a integração ensino-serviço no contexto da APS e qualificação profissional com intercâmbios multiprofissionais. $^{7-8}$

Os Programas de Residência em Saúde são resultado de uma cooperação entre os Ministérios da Saúde (MS) e da Educação, articulados para favorecer a inserção qualificada de profissionais da saúde no Sistema Único de Saúde (SUS); possuindo uma carga horária de $60 \mathrm{~h}$ semanais, com dedicação exclusiva, sendo dividido, em $80 \%$, com atividades práticas e $20 \%$ teóricas. ${ }^{9}$

Diante desse contexto de pandemia, o escopo deste estudo é revelar as experiências vivenciadas pelos residentes de enfermagem, do programa de residência multiprofissional em saúde da família, durante o período de enfrentamento à COVID-19.

\section{METODOLOGIA}

Trata-se de um relato de experiência, de abordagem descritivo-reflexiva, baseado na atuação dos residentes de Enfermagem nas Unidades de Saúde da Família (USF), que constituem os cenários de práticas do Programa de Residência Multiprofissional em Saúde da Família (PRMSF), no município de 
Cascavel/PR, no enfrentamento à pandemia da COVID-19.

Para contextualizar, a Atenção Primária em Saúde (APS) do município de Cascavel/PR está dividida em 3 Distritos Sanitários, com 49 equipes de saúde da família, correspondendo a $51,47 \%$ de cobertura da Estratégia Saúde da Família e 85,03\% de cobertura de Atenção Básica.

$\mathrm{O}$ relato envolveu a descrição das ações realizadas pelos residentes de enfermagem, no enfrentamento à COVID-19, de forma sistematizada por categorias, para o período de março a outubro de 2020, nos espaços de desenvolvimento das práticas da enfermagem, sendo: USF Presidente, USF Pioneiros Catarinense e USF Santo Onofre.

Para tanto, esse relato de experiência foi sistematizado por categorias temáticas, que foram desenvolvidas e vivenciadas pelos residentes nos cenários de prática da ESF.

Nesses territórios, as equipes das USFs eram compostas pelos profissionais da equipe mínima, preconizada pela Política Nacional de Atenção Básica (PNAB): (I) médico generalista, ou especialista em Saúde da Família, ou médico de Família e Comunidade; (II) enfermeiro generalista ou especialista em Saúde da Família; (III) auxiliar ou técnico de enfermagem; e (IV) agentes comunitários de saúde. Podem ser acrescentados, a essa composição, os profissionais de Saúde Bucal: cirurgião-dentista generalista ou especialista em Saúde da Família, auxiliar e/ou técnico em Saúde Bucal. ${ }^{6}$

Dentre os profissionais de saúde, envolvidos no enfrentamento dessa emergência sanitária, encontram-se os residentes do PRMSF, os quais são profissionais das seguintes categorias: Enfermagem, Odontologia e Serviço Social.

Ainda salientar que, para a execução deste trabalho, foram atendidas as normas dispostas nas Resoluções 466/12 e 510/2016, do Conselho Nacional de Saúde (CNS), não sendo submetido ao Comitê de Ética em Pesquisa (CEP), visto que se trata de um relato de experiência reflexivo. ${ }^{10-11}$

\section{RESULTADOS E DISCUSSÕES}

Relato de Experiência

Os residentes de enfermagem, juntamente com os preceptores e tutores, propõem, continuamente, possibilidades de melhorias para os cenários em que estão inseridos, de forma que a elaboração e reorganização de processos de trabalho possam ser adotadas nas práticas profissionais pelos servidores das Unidades de Saúde.

Com a declaração da pandemia, os profissionais e os residentes precisaram se reorganizar no processo de formação e se adaptar a essa nova realidade, imposta pela pandemia. Assim, foi essencial a execução de ações de promoção de saúde, que visassem ao enfrentamento do Coronavírus, sem desassistir os usuários do Sistema Único de Saúde (SUS) da área de abrangência da Unidade de Saúde.

A pandemia, relativa ao Coronavírus, estendeu-se por todo o mundo e, diante disso, desde março de 2020, a população de Cascavel, situada na região oeste do estado do Paraná, bem como os profissionais de saúde, vivenciaram o enfrentamento dessa pandemia.

Como integrantes dessa equipe, faz parte, neste relato, a experiência dos residentes de enfermagem, em processo de formação profissional, com base na especialização lato Sensu em Saúde da Família, na modalidade de Residência Multiprofissional.

Para tanto, esse relato de experiência encontra-se sistematizado por categorias temáticas, que foram desenvolvidas e vivenciadas pelos residentes nos cenários de prática da ESF.

\section{Reorganização da Atenção Primária em Saúde para o enfrentamento ao Coronavírus no município de Cascavel/PR}

Com a declaração da pandemia, no Município de Cascavel/PR, em março de 2020, os profissionais da APS foram surpreendidos por novas demandas, protocolos e fluxos. Diante disso, o grande desafio foi a reorganização da Atenção Primária, para que pudéssemos efetivar continuidade de assistência à população; por ser a APS/ESF porta de entrada do SUS, foi necessário reinventar processos de trabalho para fortalecer as redes de apoio. 
Em um primeiro momento, quando se deflagrou a pandemia como comunitária, houve o fechamento das unidades de saúde, para reordenação do território. Posteriormente, as Unidades de Saúde foram reabertas, nos distritos sanitários, com referências para atendimentos aos usuários, classificados como pacientes crônicos e gestantes, enquanto outras unidades de saúde seriam referência no atendimento ao paciente suspeito ou confirmado de Coronavírus. Devido a essa reorganização dos fluxos de atendimentos, foi necessária a suspensão de algumas atividades, para evitar aglomeração e proteger as pessoas de maior grau de risco; nesse primeiro momento, foram suspensas algumas ações, realizadas nas USFs, a saber, a coleta de citologia oncótica, saúde bucal, acompanhamento à criança sadia (Crescimento e Desenvolvimento), testes rápidos para o vírus da imunodeficiência humana (HIV), Sífilis, Hepatite B e Hepatite $\mathrm{C}$, exceto para as gestantes ou alguma urgência.

A Secretaria de saúde do município de Cascavel, com base na situação epidemiológica do país, implantou o serviço de Call Center, para orientação, triagem, monitoramento e notificação dos casos suspeitos de COVID-19. Portanto, os profissionais foram capacitados para identificar o curso clínico da infecção e realocados para auxiliar o telemonitoramento, incluindo-se, nesse contexto, os residentes de enfermagem. $\mathrm{O}$ atendimento à população ocorreu por meio de números de telefones, divulgados amplamente nas mídias sociais, para a população em geral.

O acompanhamento das pessoas atendidas com suspeitas de COVID-19 passou a ser realizado com base em teleatendimentos diários, registrados em uma planilha do google drive, elaborada por uma residente do serviço social do mesmo programa.

Outro ponto interessante e que deve ser ressaltado é em relação aos fluxos internos a que cada unidade teve que se adaptar, considerando a quantidade de profissionais que permaneceram na equipe, muitas vezes, não correspondendo à equipe mínima, preconizada pela Política Nacional de Atenção Básica (PNAB).
Relato de Experiência

A equipe, como um todo, teve que se readequar aos seus "papéis", em muitas situações, com a necessidade de exercer atividades que não estavam descritas nos editais do concurso, mas que, no cenário epidemiológico atual, se fizeram necessárias para efetivar continuidade à assistência e aos serviços de saúde. Destaca-se o rodízio entre os profissionais que iriam realizar o papel de "abordagem" no serviço, cargo que não existe em editais de concursos, mas que, em tempos de pandemia, foram necessários, já que consistia como o primeiro contato do paciente antes de entrar na unidade, para fins de evitar aglomerações, estabelecer ordem de chegada, aplicar álcool em gel e aferir temperatura, entre outras funções, que foram sendo estabelecidas, conforme demanda e fluxos.

Nessa perspectiva, garantir a continuidade das ações próprias, inerentes da APS, simultaneamente, com a realização dos novos cuidados para o enfrentamento da COVID-19, tornou-se um desafio para os profissionais de saúde.

\section{O desenvolvimento das ações dos Programas de Saúde da Estratégia de Saúde da Família e a pandemia do Coronavírus}

$\mathrm{O}$ enfrentamento à pandemia permitiu compreender que, mesmo na adversidade, a equipe de saúde foi capaz de se reinventar e reorganizar ações dos programas para garantir a atenção à saúde, tanto aos usuários, como as demandas pertinentes aos protocolos da Estratégia da Saúde da Família, quanto àqueles apresentados pela COVID-19.

Como medida de prevenção ao contágio da COVID-19, houve a readaptação de procedimentos e a incorporação de outras ações para que a APS funcionasse cumprindo sua missão, inclusive, com novas formas de cuidado no cotidiano das práticas profissionais desenvolvidas a distância, com a implantação do teleatendimento.

Sabemos que não é a melhor forma de acompanhar e realizar uma consulta, mas isso foi necessário, devido ao cenário em que estamos inseridos, visto que era essencial evitar que a população se deslocasse às unidades de saúde, sem deixar de prestar a 
assistência necessária. Diante desse processo de reorganização da APS, as seguintes ações foram desenvolvidas pelos residentes, tais como: alteração dos fluxos para o preenchimento da Declaração de Nascido Vivo (DNV); renovação de receitas; a telepuericultura, em que são colhidos os dados da criança, repassadas informações, bem como orientações e, quando necessário, realizado agendamento para atendimento presencial. Dessa forma, garantiu-se a oferta de ações de forma segura, de modo que não haja descontinuidade e agravamento das condições dos usuários.

Nesse sentido, é preciso lembrar que, para alguns, foram mantidos os atendimentos presenciais, assim como outras atividades de rotina, a exemplo da vacinação, que precisa ser realizada sem expor a população ao risco de contaminação. Nesses casos, as unidades de saúde estabeleceram um fluxo de atendimento diferenciado, a saber, o horário de consulta agendado via telefone; primeira abordagem ao lado externo da unidade para verificar se o paciente apresentava alguma sintomatologia gripal ou teve contato com paciente suspeito/confirmado de COVID-19; verificação da temperatura, triagem na recepção da unidade, por ser um espaço mais amplo, além de sala de isolamento para os casos suspeitos de COVID-19, a fim de que, dessa forma, não houvesse a transmissão do vírus dentro da unidade.

Nesse sentido, evidencia-se a experiência dos residentes, durante a campanha de vacinação para influenza, que ocorreu no início da pandemia, para idosos acamados em domicílio, o que possibilitou a realização de educação em saúde, preparando a comunidade sobre o atual contexto do problema de emergência de Saúde Pública.

É oportuno destacar que os residentes de enfermagem da USF estão na linha de frente, demonstrando seu compromisso ético, político e social no enfrentamento da COVID19, inseridos, como parte da equipe de saúde, além de efetivar continuidade às ações cotidianas dos programas de saúde, para garantir a atenção aos indivíduos e famílias, grupos e comunidades das suas áreas adstritas, dentro do território. Isso, com o objetivo de propiciar a formação proposta pelo programa de residência multiprofissional.

Relato de Experiência

O residente enfermeiro e suas contribuições para as mudanças do processo de trabalho nas Unidades de Saúde

Inicialmente, como não existiam casos de COVID-19, em Cascavel PR, o programa de residência iniciou suas atividades acadêmicas, conforme cronograma planejado. Portanto, os residentes foram direcionados às unidades de saúde da família, juntamente com os preceptores, para conhecimento da rotina, território, agenda e fluxos das unidades.

Com a deflagração da pandemia, nenhuma atividade prática dos residentes foi suspensa, apesar das medidas de isolamento social determinadas pelo governo. As atividades práticas foram mantidas por serem essenciais para a população e para o processo de formação dos residentes, o que seguiu em consonância com as orientações estabelecidas pelo Ministério da Saúde e da Educação.

Ficou evidente a importância do papel do residente enquanto componente da equipe, da importância para o cuidado da população, considerando que houve déficit de profissionais na assistência, em virtude de afastamentos por estarem classificados como grupos de risco. Essa experiência proporcionou aos residentes que atuassem como enfermeiros, tanto na assistência direta quanto no gerenciamento das atividades dos serviços de saúde.

Por meio da contribuição para o eixo da vigilância em saúde, os residentes atuam na parte de notificação, detecção e acompanhamento dos casos suspeitos/confirmados de Covid-19. As informações obtidas no monitoramento são registradas em prontuário, o que inclui o acompanhamento dos sintomas, evolução do quadro, investigação do surgimento de sintomas nos contatos, a fim de reforçar as orientações quanto ao isolamento domiciliar.

No enfrentamento da COVID-19, os residentes de enfermagem têm exercido papel relevante para a prevenção e controle de doenças, de maneira que colaboram, 
estrategicamente, para a redução do risco de transmissão da doença, a partir do diagnóstico precoce, por meio de acompanhamento e monitoramento do indivíduo, família e comunidade.

Diante disso, os programas multiprofissionais de residência em saúde da família tornam-se um fator de mudança de paradigmas à formação com ênfase à qualidade da assistência, no ensino e na pesquisa.

\section{CONSIDERAÇÕES FINAIS}

A proposta da reorganização dos processos de trabalho, na Atenção Primária, tem permitido o fortalecimento das ações de enfrentamento, durante a pandemia, sem, contudo, perder a essência do cuidado preconizado.

Diante desse cenário, a enfermagem se destaca, no desenvolvimento de ações em saúde, com vistas a reorganizar estratégias conjuntas com a equipe de saúde, para atendimento às necessidades e demandas da população da sua área de abrangência.

Nesse sentido, a reestruturação de espaços, bem como as estratégias implantadas e implementadas para a organização de protocolos e fluxos, que envolvem os saberes da equipe multiprofissional e interdisciplinar, tornam-se um diferencial, garantindo a continuidade das ações inerentes da APS e, paralelamente, a realização dos novos cuidados para o enfrentamento da COVID-19. Sendo assim, os residentes contribuem significativamente, possibilitando a integração de ensino, serviço e comunidade.

Dessa forma, as articulações, desenvolvidas pelos residentes de enfermagem, oportunizaram o relacionamento entre as diferentes áreas profissionais da saúde; com a pandemia, foi possível ampliar a visão acerca do cuidado, identificando a importância das outras categorias profissionais, de maneira a possibilitar a socialização do conhecimento apreendido no contexto da pandemia da COVID-19. Diante disso, tais profissionais são agentes disseminadores de informações nos diversos espaços do território.
Relato de Experiência

Nessa perspectiva, espera-se que a experiência descrita possa provocar reflexões sobre a importância da formação profissional na modalidade de residência multiprofissional, no contexto da APS, bem como as suas contribuições na atenção à saúde, visando aprimorar os processos de formação e qualificação dos profissionais de saúde, para que possibilitem mudanças e fortalecimento do Sistema Único de Saúde (SUS).

\section{REFERÊNCIAS}

1. Ministério da Saúde (BR). Secretaria de Atenção Primária À Saúde (SAPS). Protocolo de Manejo Clínico do Coronavírus para Atenção Primária. Brasília: MS; 2020. [online] [acesso em 2020 Out 20]. Disponível em:

https://saude.rs.gov.br/upload/arquivos/20200 4/14140606-4-ms-protocolomanejo-apsver07abril.pdf.

2. Ministério da Saúde (BR). Secretaria da ciência, tecnologia, inovação e insumos estratégicos em saúde. Diretrizes para diagnóstico e tratamento da COVID 19. Brasília: MS; 2020. [online] [acesso em 2020 Out 8]. Disponível em:

https://saude.rs.gov.br/upload/arquivos/20200 4/14140600-2-ms-diretrizes-covid-v2-9-4.pdf.

3. Ministério da Saúde (BR). Portaria $\mathrm{N}^{\circ} 188$, de 03 de março de 2020: declara Emergência em Saúde Pública de importância Nacional (ESPIN) em decorrência da Infecção Humana pelo novo Coronavírus (2019-nCoV). Diário Oficial da União; 2020c. [online] [acesso em 2020 Out 8]. Disponível em:

https://www.in.gov.br/en/web/dou/-/portarian-188-de-3-de-fevereiro-de-2020-241408388.

4. Ministério da Saúde (BR). Portaria $\mathrm{N}^{\circ} 454$, de 20 de março de 2020: declara, em todo o território nacional, o estado de transmissão comunitária do coronavírus (covid-19). Brasília: MS, 2020d. [online]. [acesso em 2020 Dez 19]. Disponível em: https://www.in.gov.br/en/web/dou/-/portarian-454-de-20-de-marco-de-2020-249091587. 
Relato de Experiência

residencia-medica-e-residencia-

5. Brasil. Lei No 13.979, de 6 de Fevereiro de 2020. Dispõe sobre as medidas para enfrentamento da emergência de saúde pública de importância internacional decorrente do coronavírus responsável pelo surto de 2019. Brasília, DF, 2020e. [online] [acesso em 2020 Out 8]. Disponível em: https://www.in.gov.br/en/web/dou/-/lei-n13.979-de-6-de-fevereiro-de-2020242078735 .

6. Ministério da Saúde (BR). Secretaria de Atenção à Saúde. Portaria No 2.436, de 21 de setembro de 2017: aprova a Política Nacional de Atenção Básica, estabelecendo a revisão de diretrizes para a organização da Atenção Básica, no âmbito do Sistema Único de Saúde (SUS). Brasília: MS; 2017. [online] [acesso em 2020 Out 8]. Disponível em: https://bvsms.saude.gov.br/bvs/saudelegis/gm /2017/prt2436_22_09_2017.html.

7. Nakahata, KS. Atividades desenvolvidas por enfermeiros em programa de residência multiprofissional. [dissertação]. São Paulo: Universidade Santo Amaro/Mestrado em Ciências da Saúde; 2017. [online]. [acesso em 2020 Dez 19]. Disponível em:

http://dspace.unisa.br/bitstream/handle/12345 6789/393/Karisa\%20Santiago\%20Nakahata\% $20-$

$\% 202017$.pdfok.pdf?sequence $=1 \&$ isAllowed $=\mathrm{y}$.

8. Cascavel. Secretaria Municipal de Saúde, Escola de Saúde Pública Municipal. Projeto Político Pedagógico do Programa de Residência Multiprofissional em Saúde da Família. Cascavel; 2018.

9. Brasil. Lei No 6.988, de 10 de maio de 2019. Institui os Programas de Residência Médica e Residência Multiprofissional em Área de Saúde e Dá Outras Providências. Cascavel, PR, 2019. [online] [acesso em 2020 Out 8]. Disponível em: https://leismunicipais.com.br/a1/pr/c/cascavel /lei-ordinaria/2019/698/6988/lei-ordinaria-n 6988-2019-institui-os-programas-de- multiprofissional-em-area-de-saude-e-daoutras-providencias.

10. Ministério da Saúde (BR), Conselho Nacional de Saúde, Comissão Nacional de Ética em pesquisa. Resolução No 466, de 12 de dezembro de 2012: dispõe sobre as diretrizes e normas regulamentadoras de pesquisas envolvendo seres humanos. Brasília: MS; 2012. [online] [acesso em 2020 Out 8]. Disponível em: https://bvsms.saude.gov.br/bvs/saudelegis/cns /2013/res0466_12_12_2012.html.

11. Ministério da Saúde (BR), Conselho Nacional de Saúde, Comissão Nacional de Ética em pesquisa. Resolução No 510, de 07 de abril de 2016: dispõe sobre as normas aplicáveis a pesquisas em Ciências Humanas e Sociais cujos procedimentos metodológicos envolvam a utilização de dados diretamente obtidos com os participantes ou de informações identificáveis ou que possam acarretar riscos maiores do que os existentes na vida cotidiana, na forma definida nesta Resolução. Brasília: MS; 2016. [online] [acesso em 2020 Out 8]. Disponível em: https://www.in.gov.br/materia//asset_publisher/Kujrw0TZC2Mb/content/id/ 22917581.
Recebido em: 13.11.2020

Aprovado em: 22.12.2020 